\title{
ESPECIE NUEVA DE RHYPASMA PASCOE, 1862 (COLEOPTERA: TENEBRIONIDAE) DE REPÚBLICA DOMINICANA
}

\author{
Orlando H. Garrido y Carlos Varela ${ }^{2}$ \\ ${ }^{1}$ Calle 60 \#1706 e/e 17 y 19, C.P. 11300, Playa, La Habana, Cuba \\ ${ }^{2}$ Acuario Nacional de Cuba (ANC), Calle 1ra \#6002 e/e 60 y 62, C. P. 11300, Playa, \\ La Habana, Cuba.carlosv@acuarionacional.cu
}

\section{RESUMEN}

Se describe una especie nueva de Rhypasma de República Dominicana. Las diferencias fundamentales con las restantes especies conocidas también son presentadas.

Palabras clave: Coleoptera, Tenebrionidae, Rhypasma, especie nueva, República Dominicana.

\begin{abstract}
A new species of Rhypasma is described from the Dominican Republic. The main differences of this new species with the others known are also given.

Key words: Coleoptera, Tenebrionidae, Rhypasma, new species, Dominican Republic.

\section{INTRODUCCIÓN}

El género Rhypasma Pascoe, 1862, se halla representado en Las Antillas Mayores por una sola especie, $R$. haitianum Marcuzzi, 1953, en Haití y en Cuba (Spillman, 1961 y Marcuzzi, 1984). Las especies más cercanas en las Antillas Menores son, R. mariagratia Marcuzzi, 1953, en Bonaire y Curaçao, $R$. trinitatis Marcuzzi, 1953, en la isla de Trinidad. y $R$. venezuelenae Marcuzzi, 1953, en la isla de Guadalupe, además de otras islas ubicadas al norte de Venezuela, como Los Frailes, Los Testigos, La Orchila y Bonaire, aparte de la propia Venezuela (Marcuzzi, 1984).

Gracias a la expedición entomológica organizada a la República Dominicana por Víctor L. González, dirigida por el colega Antonio R. Pérez-Asso, se colectó una interesante muestra de insectos, entre ellos la nueva especie que a continuación se describe.
\end{abstract}

\section{OBJETIVO}

- Describir una especie nueva de insecto del género Rhypasma; Familia Tenebrionidae, Orden Coleoptera.

\section{MATERIALES Y MÉTODOS}

Se llevaron a cabo colectas en el campo en septiembre del 2004, en la provincia de Pedernales, República Dominicana. Estas se realizaron manualmente y para ello se revisó en troncos caídos, hojarasca y debajo de piedras. Los ejemplares fueron pasados a un frasco con alcohol, luego montados en alfileres entomológicos y bajo un microscopio estereoscópico se observaron los caracteres de los mismos. El espécimen estudiado y descrito se encuentra depositado en la colección de la Hacienda Paraíso, kilómetro 10, Real Anón. Ponce, Puerto Rico. 


\section{RESULTADOS \\ TAXONOMÍA \\ Género Rhypasma Pascoe, 1862}

Referencia. Pascoe, F. 1862. Notes on Coleoptera with description of new genera and species. Part I. Annals and Magasin of Natural History, 8 (4): 345-361.

\section{Rhypasma quisqueyanus sp. nov.}

Figura 1

Diagnosis. Pequeño tamaño (4.5 mm). De color negro semiopaco, con los élitros más negros que el pronoto y la cabeza. Cabeza y pronoto densamente punzados. Pronoto caliciforme, con los lados lisos, ápices puntiagudos y la base mucho más estrecha que la de los élitros, los cuales están punzados y tienen tres conspicuas carinas en cada uno.

Diagnosis. Small size (4.5 mm). Dull black, with elytra blacker than the pronotum and the head. Head and pronotum densely punctured. Caliciform pronotum with smooth lateral sides and the apexes pointed with the base much narrower than the elytra, which are punctured with three conspicuous keels on each side.

Descripción del holotipo. Cabeza semiredondeada. Densamente punzada con punciones redondas y dispersas. Genas bien discernibles con punciones similares. Ojos negros alargados que quedan alojados en una cavidad profunda. Clípeo recto y bien discernible. Antenas moniliformes, que llegan casi hasta la base del pronoto, con 11 artejos, gruesos, pilosos y prácticamente uniformes en tamaño. Partes inferiores castaño oscuro. Partes bucales del mismo color que las antenas. La región de la cabeza que linda con el pronoto lisa y lustrosa, la superior homogéneamente punzada con punciones diminutas.

Pronoto. En forma de cáliz. Base con los ápices puntiagudos y mucho más estrecha que la de los élitros. Superficie densamente punzada, con las punciones redondas y profundas, algo dispersas y con pequeñas cerdillas. Bordes laterales de un castaño más oscuro. El color castaño oscuro de la cabeza es similar al del pronoto y contrasta con el color de los élitros. Partes inferiores con la región apical lisa y de un castaño más oscuro que contrasta con el resto del pronoto. Prosternum punzado, largo y tubiforme, con la parte inferior terminando en forma de lengüeta por debajo de las coxas del primer par de patas.

\section{Escutelo. Pequeño y triangular.}

Élitros. Muy oscuros, casi negros, más oscuros que el pronoto y la cabeza. Dos veces y medio el largo del pronoto. Bordes paralelos en casi toda su extensión, menos en el tercio apical donde se van estrechando paulatinamente. Élitros fundidos. Tres conspícuas carinas, en cada élitro, longitudinales, que llegan hasta el tercer tercio apical. Dos filas de gruesas punciones redondas y paralelas, simétricamente dispuestas entre cada carina, dichas punciones el doble de grueso que las del pronoto. Superficie opaca.

Mesosternum elevado en forma semitriangular, liso y con espaciadas cerdillas en su parte superior y numerosas punciones dispuestas simétricamente en hileras. Prosternum y Metasternum con numerosas hileras de punciones.

Epipleura con dos filas de promontorios dispuestos simétricamente. 


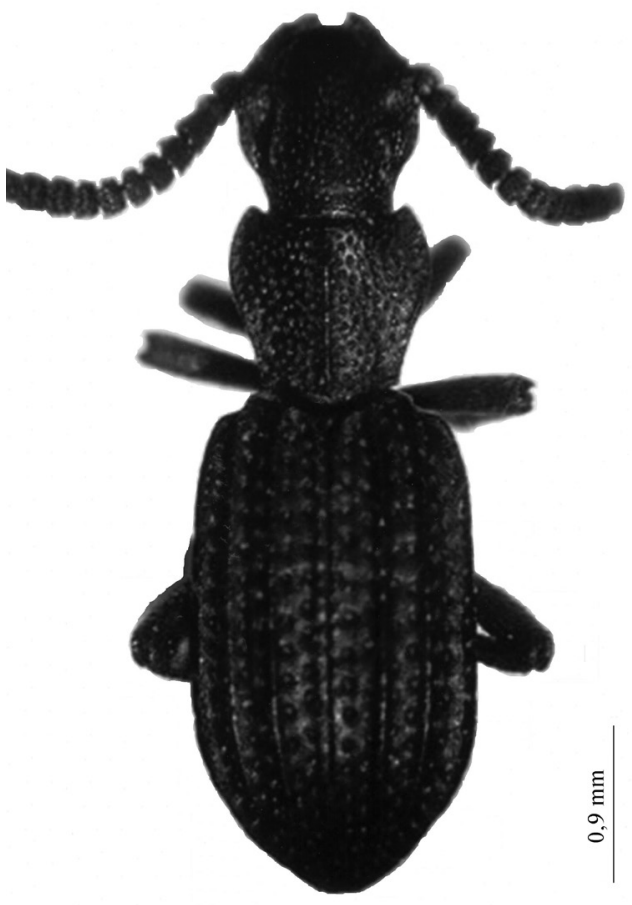

Figura 1. Rhypasma quisqueyanus sp. nov. Vista dorsal del holotipo.

Miembros. Fémures y tibias rectos, los primeros el doble de gruesos de los segundos. Tibias pilosas con un par de espinas poco conspicuas en la coyuntura con los tarsos, los cuales tienen los artejos prácticamente similares y sin pubescencia.

Tipos. Holotipo, HPPR-3876. Cueva del Guano, carretera a Pedernales, República Dominicana. Colectado el 12 de septiembre del 2004 por Antonio R. Pérez Asso. Depositado en la Colección Entomológica Hacienda Paraíso (HPPR), Hacienda Paraíso, kilómetro 10, Real Anón. Ponce, Puerto Rico.

Etimología: quisqueyanus, de Quisqueya, nombre con que se conocía La Hispaniola a la llegada de los españoles.

Comentarios. Los lados del pronoto lisos y la forma característica del mismo distinguen a Rhypasma quisqueyanus sp. nov. de las restantes especies del género. Con respecto, específicamente, a las especies de la región caribeña, en $R$. haitianum y $R$. trinitatis el máximo ancho del pronoto se encuentra en su porción media, mientras que en $R$. quisqueyanus sp. nov. se localiza en su porción superior. En $R$. venezuelenae el pronoto es trapezoidal, mientras que en $R$. quisqueyanus sp. nov. es caliciforme.

\section{LITERATURA CITADA}

Marcuzzi, G. 1984. A Catalogue of Tenebrionid beetles (Coleoptera: Heteromera) of the West Indies. Folia Entomologica Hungarica. XLV.1: 69-108.

Pascoe, F. 1862. Notes on Coleoptera with description of new genera and species. Part I. Annals and Magasin of Natural History, 8 (4): 345-361.

Spillman, T. J. 1961. A few tenebrionids new to Cuba. Coleopterists's Bulletin, 15: 4. 